\title{
Kasuistik: Systemische Enthesitis unter Lithiumerhaltungstherapie wegen einer Bipolaren Störung
}

\section{Case-Report: Lithium-related systemic Enthesitis}

\section{Anamnese}

Wegen eines seit Monaten bestehenden dumpfen Schmerzes im linken distalen Unterschenkel und - weniger ausgeprägt seit einigen Wochen auch rechts suchte der 62-jährige Mann eine Rheumaambulanz auf. Die Schmerzen hätten nach einer angiologischen Untersuchung beider Unterschenkel wegen einer Varikosis begonnen und sprachen zunächst gut auf Ibuprofen (200 mg über 10 Stunden) an, doch dann wurde ein zusätzliches Morphinpflaster (12,5 mg/3 Tage) notwendig.

Anamnestisch war noch zu erfahren, dass der Patient wegen einer in der Vorgeschichte diagnostizierten bipolaren Störung seit 11 Jahren eine regelmäßige laborkontrollierte Lithiumtherapie hatte, unter der er beschwerdefrei sei. Vor 8 Jahren sei dann eine Psoriasis capitis aufgetreten, die erfolgreich dermatologisch behandelt wurde.

\section{Klinischer Befund}

Klinisch bestand eine schmerzhafte Weichteilschwellung oberhalb des Außenknöchels links, ohne Rötung bei leichter Unterschenkelvarikosis beiderseits. Im Ultraschall ergab sich der Verdacht auf ein verkalktes periossäres Hämatom. Die Finger zeigten eine mäßige Polyarthrose, aber keine Daktylitis, die Bauchhaut einzelne Psoriasiseffloreszenzen.

Labor: BKS in der 1. Stunde $61 \mathrm{~mm}$, CRP $15 \mathrm{mg} / \mathrm{l}$, Elektrolyte und Nierenwerte im Normbereich. ANA Titer 1 : 1280 (schwach positiv); doppelsträngige DNS negativ.

\section{Bildgebung}

Die Projektionsradiogramme, das CT und die Ganzkörper-Skelettszintigraphie ( $\triangleright$ Abb. 1a-e) zeigen knöcherne, wabig strukturierte Auflagerungen, die vom Periost der distalen linken Fibulametadiaphyse ausgehen. Vom Gesamtaspekt her ist dieser Befund gut mit der Diagnose einer systemischen Enthesitis (Spondarthritis) bei Psoriasis zu vereinbaren, auch wenn die für dieses Krankheitsbild sonst so typische Kombination von Osteodestruktion und Osteoproliferation nicht voll ausgeprägt ist. Der Begriff „systemische Enthesitis“ leitet sich von dem systemischen Auftreten destruktiv-proliferativer Skelettveränderungen an allen Übergängen vom Periost und von MuskelSehnen- und Gelenkkapselansätzen - den Enthesen [1] - am Knochen ab. Er ersetzt den konventionellen Begriff „seronegative Spond(ylo)arthritis“ [2]. Systemische Enthesitiden gibt es vor allem bei Psoriasis und Pustulosis palmoplantaris, reaktiv z.B. bei M. Reiter, idiopathisch vor allem bei jüngeren Männern (sog. M. Bechterew/ankylosierende Spondarthritis) und bei entzündlichen Darmerkrankungen. In der Regel lässt sich nichtinvasiv die Diagnose aus den anamnestischen, klinischen und radiologischen Befunden stellen. 


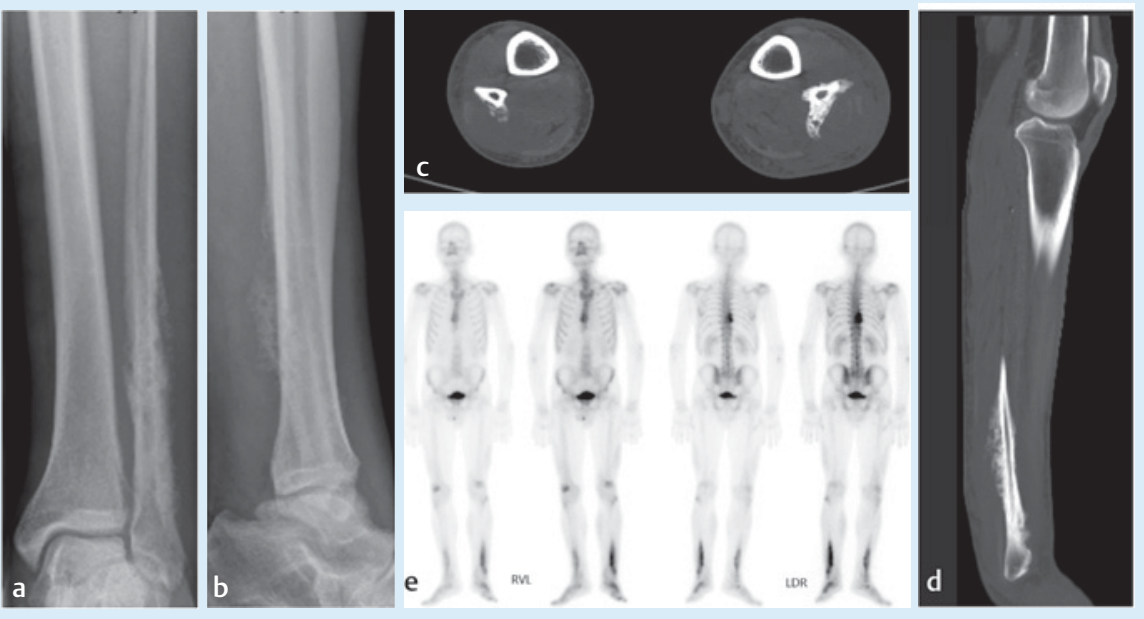

- Abb. 1a-e: Pelzig-wabige Verknöcherungen, die der distalen linken Fibuladiametaphyse aufsitzen (a, b). Die CT-Aufnahmen (c, d) beweisen, dass die darunter gelegene Kompakta solide ist. Auf der Gegenseite überraschenderweise ähnliche, aber weniger ausgeprägte Periostverknöcherungen. Das Ganzkörperskelettszintigramm (e) zeigt in den distalen Fibulae - links stärker als rechts - pathologische Aktivitätseinlagerungen. Ebenfalls überraschend findet sich eine starke exzentrische Tracereinlagerung in BWK 8 und 9. Radiologisch stellten sich dort ausgeprägte hyperostotische Veränderungen dar, wie sie typisch für eine systemische Enthesits (sog. seronegative Spondarthritis) sind. Insgesamt betrachtet lässt sich unter Berücksichtigung der psoriatischen Veränderungen an der Kopf-und Bauchhaut der Schluss ziehen, dass der Patient eine psoriatische systemische Enthesitis hat. Damit wäre eine histologische Abklärung aus radiologischer Sicht nicht notwendig gewesen. a-c Plain radiographs of the left fibula show hairy and bubble-forming ossifications at the distal metaphyseal bone surface (a, b), without interruption of the underlying corticalis as CT sections reveal (c, d). The right-sided fibula shows similar lesions, albeit in a lesser degree. Accordingly, bone scintigraphy shows hot spots at both distal fibulae, although in a different activity. Additionally, high activity was displayed at the 8th and 9th thoracic vertebra in a somewhat excentric configuration, caused by hyperostotic spurs as plain radiographs reveal. This is a highly characteritic finding in systemic enthesitis (so called seronegative spondylarthritis). Taken together the patients history, the skeletal findings and the psoriatic lesions, from a radiologic perspective the diagnosis of a systemic enthesitis in lithium related psoriasis can be reasonable established - without the need of a bioptic confirmation.

Unter der externen Verdachtsdiagnose „Ossifizierende Periostitis“ und zum Tumorausschluss erfolgte eine offene Biopsie am linken Unterschenkel mit Entnahme von Gewebeproben aus fibrös veränderter Muskulatur und Verknöcherungen von der Oberfläche der distalen Fibulametaphyse beugeseitig und medial.

\section{Histopathologie}

Histologisch fand sich ein fibrös verbreitertes Periost mit unregelmäßiger metaplastischer Knochenbildung und bereits fokaler Ausreifung zu lamellärem Knochen sowie vereinzelte knorpelige Matrix. Eine geringe perivasale lymphozytäre Infiltration war ebenfalls nachweisbar. Die aufliegende Skelettmuskulatur war in den Prozess einbezogen, mit Einschluss stehengebliebener Muskelfasern im fibrösen Gewebe ( Abb. 2 a-d). Immunhistologisch fiel die MDM2-Reaktion negativ aus. Molekularpathologisch (FISH) fand sich keine
Amplifikation des MDM2-Gens. Zusammenfassend lag somit rein histomorphologisch eine chronische ossifizierende Periostitis mit Einbeziehung der angrenzenden Skelettmuskulatur vor.

\section{Diagnose in der Zusammenschau aller klinischen, radiologischen und histopathologischen Befunde}

Systemische Enthesitis der distalen Fibulae links stärker als rechts - unter Lithiumerhaltungstherapie mit wenig florider Psoriasis.

\section{Diskussion}

Die Enthesen sind spezialisierte Übergangszonen in der Insertion von Muskelsehnen und Gelenkkapseln an der Knochenoberfläche und dienen der Kraftübertragung [3]. Mehr als 100 derartige Strukturen liegen im menschlichen Körper vor. Enthesitiden unterscheiden sich fundamental von der Synovialitis. Während bei der Enthesitis eine extraartikuläre Entzündung des
Bindegewebes und Knochens vorliegt, ist bei der Synovialitis die intraartikuläre Synovialmembran betroffen $[4,5]$.

Histologisch unterscheidet man bei den Enthesen den fibrösen vom fibrokartilaginären Typ. Bei ersterer, der selteneren und typischerweise metadiaphysär gelegenen Form, treten Kollagenfasern vom Sehnengewebe direkt in das Periost oder den mineralisierten Knochen über, bei der zweiten - der häufigeren und typischerweise apo-und metaphysär lokalisierten Form ist eine Schicht von avaskulärem Faserknorpel intermediär zwischen Bindegewebe und Knochen ausgebildet [1].

Im vorliegenden Fall ist die Enthese der Mm. flexor digitorum und hallucis longus in der distalen Fibulametaphyse betroffen. Da die Veränderungen beiderseits vorliegen und sich ein typischer Befund an der unteren BWS fand, handelt es sich um eine systemische Enthesitis. Während der unilokulären Enthesitis (z.B. Tennis-/GolferEllenbogen) in aller Regel eine mechanische Überlastung zugrunde liegt, ist die Pathogenese der systemischen Enthesitis noch weitgehend unklar. Anscheinend ist die Grenzzone zwischen gering- oder nicht-vaskularisiertem Bindegewebe und dem gut vaskularisierten Knochen Ort der Störung. Für die Differenzialdiagnose ist die Detektion mehrerer Herde, z.B. durch eine Skelettszintigrafie, äußerst hilfreich, da solche eine Neoplasie praktisch ausschließen.

Bei der Inzisionsbiopsie kann die mikroskopische Differenzialdiagnose zwischen einer Enthesitis einerseits und einem primären low grade parossalen Osteosarkom andererseits Schwierigkeiten bereiten. Letzteres liegt definitionsgemäß an der Knochenoberfläche und tritt bevorzugt metaphysär an langen Röhrenknochen auf, auch noch im Erwachsenalter. Mikroskopische Zellatypien, Proliferationsaktivität und Invasivität können bei diesen Tumoren minimal und nur fokal ausgebildet sein. Da auch immunhistochemische und molekularpathologische Zusatzuntersuchungen (MDM2-Nachweis bzw. -Amplifikation) negativ verlaufen können, ist die Einbeziehung der klinisch-radiologischen Befunde in solchen Fällen zur Diagnosestellung unabdingbar. 


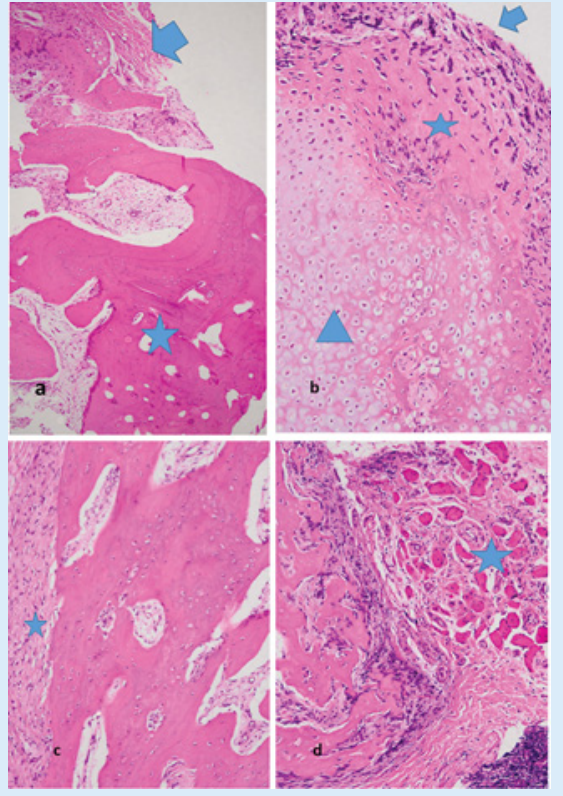

- Abb. 2 a-d: Histobioptische Befunde (HE, 10X). a) Umbau der Kortikalis $\left({ }^{*}\right)$ mit Fibrose und metaplastischer Verknöcherung im Periost $(\leftarrow)$; b) Zellreiche fibrosierende Entzündung an der Knochenoberfläche $(\leftarrow)$ mit metaplastischer Knochen $\left({ }^{*}\right)$ - und Knorpel $(\Delta)$-Bildung; c) Zellreiche Fibrose mit reaktiven Kernatypien ( ${ }^{*}$ ) und unregelmäßige Knochenstruktur können die Differenzialdiagnose zu einem Low-gradeparossalen-Osteosarkom in der Biopsie schwierig machen; d) Einbeziehung der angrenzenden Skelettmuskulatur $\left({ }^{*}\right)$ in die Enthesitis mit reaktiver lymphozytärer Entzündung in der Grenzzone können histologisch einen invasiven Prozess vortäuschen. a-d Histobioptic findings ( $\mathrm{HE}$, 10X). (a) Compacta remodelling $\left({ }^{*}\right)$ and periostal spindle cell fibrosis with metaplastic bone formation $(\leftarrow)$; b) Cellular fibrosing inflammation at the bone surface $(\leftarrow)$ with calluslike bone $\left({ }^{*}\right)$ - and cartilage $(\Delta)$-formation; c) spindle cell proliferation with reactive nuclear atypia $\left({ }^{*}\right)$ and irregular bone structure may be focally very similar to a low grade parosteal osteosarcoma. Correct assessment of the process within the limited tissue of a biopsy, exclusively on histologic grounds can become problematic; d) Inclusions of muscle parenchyma within the process with dense lymphocytic infiltrates in the border zone may mimic an infiltrative tumor. Additional clinical, imaging and scintigraphic findings may be of paramount importance to reach the correct diagnosis.
Im hier präsentierten Fall erhielt der Patient seit Jahren eine Lithiumerhaltungstherapie. Deren Nebenwirkungen sind gut erforscht; vor allem Störungen der Nierenfunktion, eine Hypothyreose und erhöhte Serumcalciumspiegel können auftreten [6, 7]. Der Patient zeigte diesbezüglich bei regelmäßigen Kontrollen keine Auffälligkeiten. Die neuropsychiatrische Wirkung von Lithium wird unter anderem seiner Eigenschaft zugeschrieben, durch eine Hemmung der Glykogensynthase- Kinase3ß (GSK-3) den kanonischen WNT/ß-catenin-Signalweg zu aktivieren, der ein hochkonservierter Signalweg für die Zellentwicklung ist [8]. Er ist entsprechend auch wichtig für verschiedenen Strukturprozesse im Gehirn [9]. Diese Kaskade stimuliert aber auch die Proliferation der Osteoblasten und deren Produktion von Osteoprotegerin (OPG) mit konsekutiver Erhöhung des OPG/RanklQuotienten und einer Verschiebung des Knochenstoffwechsels zugunsten des Knochenanbaus im Verhältnis zur osteoklastären Knochenresorption. Diesen über den WNT/ß-Catenin-Signalweg vermittelten positiven Effekt auf die Knochenbildung durch Lithium - sowohl bei einer systemischen Supplementation als auch bei einer lokalen Applikation - wurde experimentell in vivo und in vitro bestätigt [10-12]. Zurzeit wird in einer Phase-II-Studie untersucht, ob eine niedrig dosierte orale Lithiumapplikation eine positive Wirkung auf die Knochenfrakturheilung hat [13].

Das Risiko, dass es unter einer Lithiumtherapie zu einer Exazerbation einer vorbestehenden Psoriasis kommen kann, ist klinisch schon lange bekannt [14]. Lithium kann jedoch auch eine Psoriasis einschließlich einer komplizierenden Enthesitis induzieren $[15,16]$, wobei die Latenzperiode nach Beginn der Lithiumtherapie im ersten Fall mehrere Wochen und im zweiten meistens viele Monate betragen kann. Da sich anamnestisch bei unserem Patienten die Psoriasis capitis erst Jahre nach Beginn der Lithiumtherapie entwickelt hat, kann man annehmen, dass sie durch Lithium induziert ist. 2012 konnten Hampton et al. [17] in einer In-vitro-Studie nachweisen, dass diese dermatologische Komplikation der Lithiumtherapie auch auf die GSK-3-hemmende pharmakologische Wirkung des Lithium zurückzuführen sein dürfte.
Wie oben erwähnt, ist das typische pathoanatomische Element einer systemischen Enthesitis die simultan bestehende Osteodestruktion und Osteoproliferation. Die Frage, weshalb im vorliegenden Fall einer systemischen psoriatischen Enthesitis mit typischem Befallsmuster die destruktive Komponente an den Fibulae radiologisch fehlt, könnte durch die offensichtlich dominierende osteoproliferative Wirkung des Lithiums beantwortet werden.

Wegen der sonstigen guten Toleranz der Therapie mit stabiler psychischer Verfassung einerseits und der nur geringen Aktivität der auf die Kopfhaut beschränkten Psoriasis andererseits wurde beschlossen, die Lithiumtherapie weiterzuführen. Wegen der Enthesitis der Fibula beiderseits wurde außerdem eine niedrig dosierte Methotrexattherapie mit - bei Bedarf zusätzlicher Schmerztherapie eingeleitet.

\section{Zusammenfassung}

Vorgestellt wird ein Fall einer systemischen Enthesitis bei einem 62-jährigen Mann mit langjähriger, gut vertragener Lithiumtherapie wegen einer Bipolaren Störung in der Jugend und darunter aufgetretenen Psoriasis capitis. Die Algorithmen der korrekten Diagnosestellung in Klinik, Bildgebung und Histobiopsie werden aufgezeigt. Die Pathophysiologie der systemischen Enthesitis mit Bezug zur bekannten pharmakologischen Lithiumwirkung auf die Osteoblastenaktivität und Knochenbildung über die Beeinflussung des WNT/ß-Catenin-Signalwegs werden dargestellt.

\section{Schlüsselwörter:}

Enthesitis, Lithiumtherapie, Osteoblastenaktivierung, WNT/ß-Catenin Signalweg, parosteales Osteosarkom

\section{Abstract}

We present a case of a systemic enthesitis in a 62-year old man under a maintenance lithium therapy since an episode of a bipolar disorder in his adolescence and with a psoriasis capitis of low activity since years. The pathophysiology of the enthesitis is discussed in relation to the pharmacology of lithium and its well-known induction of osteoblastic activity and bone formation via the WNT/ß-catenin pathway. 


\section{Keywords:}

Enthesitis, lithium maintenance therapy; osteoblasts; WNT/ß-catenin pathway; parosteal osteosarcoma

\section{Autorinnen / Autoren}

\section{Helmut Ostertag, Helmut*}

*Pathologisches Institut Klinikum Region Hannover, Nordstadtkrankenhaus Hannover

\section{Jürgen Freyschmidt, Jürgen§}

$\S$ Klinikum Bremen Beratungsstelle und Referenzzentrum für Osteoradiologie Bremen

\section{Pia Freyschmidt Pia ${ }^{\circ}$}

- Dermatologische Praxis Schwalmstadt-Treysa

\section{Korrespondenzadresse}

Prof. Dr. med. Helmut Ostertag

Pathologisches Institut Krankenhaus Nordstadt Haltenhoffstr 41

30167 Hannover

E-Mail: Helmut.Ostertag@krh.eu

\section{Literatur}

[1] Apostolakos ], Durant TJS, Dwyer CR et al. The enthesis: A review of the tendon-to-bone insertion. Muscels Ligaments Tendons J 2014; 4: $333-342$
[2] Freyschmidt J, Freyschmidt P. Das EnthesitisKonzept - ein Paradigmenwechsel in der radiologischen Diagnostik seronegativer Spondyloarthritiden. Radiologie up2 date 2018: $35-48$

[3] Jakob F, Müller-Deubert S, Ebert R et al. Interaktion zwischen Muskel und Knochen - Ein Wechselspiel zwischen Physik und Biologie. Osteologie 2020; 29: 111-122

[4] Schett G, Lories RJ, D’Agostino M-A et al. Enthesitis. From pathophysiology to treatment. Nat Rev Rheumatol 2017; 13: 731-741

[5] Kehl AS, Corr M, Weisman MH. Review. Enthesitis: New Insights Into Pathogenesis, Diagnostic Modalities, and Treatment. Arthritis Rheumatology (Hoboken, N J) 2016; 68: 312-322

[6] McKnight RF, Adida M, Budge K et al. Lithium toxicity profile. Syst Rev Meta-Anal. Lancet 2012; 379: 721-728

[7] Albert U, Cori DD, Blengino G et al. Trattamento con litio e potenziali effetti collaterali a lungo termine. Una revisione sistematica della letteratura. Riv Psichiatr 2014; 49: $12-$ 21

[8] Hedgepeth CM, Conrad L], Zhang J et al. Activation of the Wnt signaling pathway. A molecular mechanism for lithium action. Dev Biol 1997; 185: 82-91

[9] Alda M Lithium in the treatment of bipolar disorder. Pharmacology and pharmacogenetics. Mol Psychiatry 2015; 20: 661-670

[10] Huang T-B, Li Y-Z, Yu K et al. Effect of the Wnt signal-RANKL/OPG axis on the enhanced osteogenic integration of a lithium incorpora- ted surface. Biomater Sci 2019; 7: 11011116

[11] Kurgan N, Bott KN, Helmeczi WE et al. Low dose lithium supplementation activates Wnt/ $\beta$-catenin signalling and increases bone OPG/ RANKL ratio in mice. Biochem Biophys Res Commun 2019; 511: 394-397

[12] Vachhani K, Whyne CM, Schaffer A et al. Perceptions of using lithium in fracture management. A survey of orthopaedic surgeons, fracture patients and the general public. BMC Musculoskelet Disord 2019; 20: 389

[13] Nam D, Balasuberamaniam P, Milner K et al. Lithium for Fracture Treatment (LiFT). A double-blind randomised control trial protocol. BMJ Open 2020; 10: e031545

[14] Bork K. Arzneimittelnebenwirkungen an der Haut. Klinik, Diagnostik zur Erkennung der auslösenden Medikamente, Pathogenese, Therapie; mit 90 Tabellen S. vol. 2. Aufl. Stuttgart: Schattauer; 1999: 324-328

[15] Lester EB, Cook DL, Friesing GW. Psoriasiform drug eruptions and drugs that flare psoriasis. In: Hall JC, Hall BJ, eds. Cutaneous drug eruptions. Psoriasiform drug eruptions and drugs that flare psoriasis. London: Springer; 2015: 141-155

[16] Balak DM, Hajdarbegovic E. Drug-induced psoriasis. Clinical perspectives. Psoriasis (Auckl) 2017; 7: 87-94

[17] Hampton PJ, Jans R, Flockhart RJ et al. Lithium regulates keratinocyte proliferation via glycogen synthase kinase 3 and NFAT2 (nuclear factor of activated T Cells 2). J Cell Physiol 2012; 227: 1529-1537 